\title{
Principal component analysis of performance test traits in Hungarian Sporthorse mares
}

\begin{abstract}
The analysis utilized data on performance traits recorded between 1993 and 2004 on 3- and 4-year-old Hungarian Sporthorse mares. Traits were categorized in three groups, chosen to describe conformation, free jumping and movement. Low to moderate correlations were found among traits within each of those groups. There were high correlations between type and frame for both ages; and within free jumping performance traits, jumping style and jumping ability were highly correlated as well. In principal component analyses of test results for 3- and 4-year-old mares, 9 factors (ratio of variance $=80.935$ ) and 7 factors (ratio of variance $=74.115$ ) were identified, respectively. Dendograms based upon cluster analysis verified the separation of trait groups. The trait of "impulsion in elasticity of movement" could be assigned to movement traits in 3-year-old; but as a probable consequence of training, it could be assigned to conformation traits, especially to overall impression, when horses were 4-year-old.
\end{abstract}

Key Words: principal component analysis, performance traits, Sporthorse mare, Hungary

\section{Zusammenfassung}

Titel der Arbeit: Hauptkomponentenanalyse der Eigenleistungsprüfung von Stuten einer ungarischen Sportpferdepopulation

Die Analyse basiert auf den Ergebnissen der Eigenleistungsprüfung von drei- bzw. vierjährigen Stuten einer ungarischen Sportpferdepopulation aus den Jahren 1993 bis 2004. Die Merkmale werden den drei Merkmalsgruppen „Typ und Körperform“, „freies Springverhalten“ sowie „Gangverhalten“ zugeordnet. Die Datenanalyse zeigt niedrige bis mittlere Korrelationen innerhalb der Merkmalsgruppen. In beiden Altersgruppen bestehen enge Korrelationen zwischen dem Typ und dem Rahmen sowie den freien Springmerkmalen, wie dem Springstil und dem Springvermögen. Für die Hauptkomponentenanalyse der dreijährigen Stuten werden 9 (Ratio der Varianz=80,935), bei den vierjährigen Stuten 7 Faktoren (Ratio der Varianz=74,115) bestimmt. Die auf den Ergebnissen der Clusteranalyse beruhenden Dendogramme zeigen die Trennung der einzelnen Merkmalsgruppen. Danach kann bei den dreijährigen Stuten das Merkmal „Impulsivität und Elastizität der Bewegung“ aus der Gruppe Exterieurbeurteilung den Bewegungsmerkmalen, jedoch bei den vierjährigen, als vermutliche Konsequenz aus dem Training, der ersten Merkmalsgruppe zugeordnet werden.

Schlüsselwörter: Hauptkomponentenanalyse, Eigenleistungsprüfung, Sportpferde, Stuten, Ungarn

\section{Introduction}

Conformation traits in sport horses are not difficult to define and evaluate, but satisfactory identification of traits that influence performance and safety during sporting events has yet to be adequately achieved. Such performance has mostly been defined by general aptitude to movement, i.e., the regularity, clear rhythm and springiness of basic strides, as well as the ability to move in a naturally balanced manner (MIHÓK, 2005).

Analysis of variance and product moment correlations are widely used to characterize phenotypic and genetic relationships among traits in a horse breeding program (PETZOLD, 1991; DIETL et al., 2004, 2005). Although used less frequently, principal 
component analysis is a valuable refinement. It examines covariation among any number of variables, allowing them to be assigned to groups that characterize different properties of the system, to quantify variation attributable to each such group and to identify relationships among traits within groups. Explanation and identification of trait groups can be used for development and verification of performance test and genetic evaluation systems.

There is a substantial German literature on genetic parameters of various performance traits of the horse (BUGISLAUS et al., 2004). For clear understanding of and the improvement of horse breeding programs, clear definitions of performance test traits are required. International review of these traits has been reported by KOENEN (2002). Objectivity and effectiveness of horse performance test programs were evaluated by SCHWARK et al. (1992) and LENGERKEN and SCHWARK (2002).

The Hungarian Sporthorse is a noble riding and harness horse with a good constitution and an aesthetic and functional conformation (MSLT, 2000). The overall objective of the present work is to improve performance testing and genetic evaluation procedures for Hungarian Sporthorse mares. In this report, we use principal component analysis to assign traits recorded on 3- and 4-year-old mares to classes representing conformation, free jumping ability and movement and to determine whether such classes are age dependent

\section{Materials and methods}

Traits evaluated on each mare in the national performance recording system are meant to evaluate conformation, free jumping and movement analysis as follows:

Conformation traits: type, head, neck, saddle region, frame, forelimbs, hindlimbs, regulation of movement, impulsion and elasticity of movement, overall impression.

Free jumping: jumping style, jumping ability-sense of distance; observation during training.

Movement analysis: walk, trot, canter, overall impression, test rider's score.

Scores for free jumping and movement analysis traits are on a 0-10 scale. Conformation traits are judged on different scales since 1. January 2000. Weighted traits are neck, forelimbs, hindlimbs and impulsion and elasticity of movement with scores between 0 and 12 . Type (0-6), head $(0-8)$ and frame $(0-8)$ are judged with less weight. Before 2000, the conformation traits were scored in the range of 0 and 10 . Observation during training, a free jumping trait, is scored by the trainer based upon cumulative impressions throughout training. The test rider's score is given by the test rider based upon subjective judgment of the rideability of the horse. Other traits are scored by an invited judge. The final score for the mare test is the sum of the mean of the conformation score, the mean of free jumping performance scores and the mean of movement analysis scores multiplied by 1, 1.5 and 2, respectively (MSLT, 2000).

Data used for the analysis were supplied by MSLT. Test records of 3- and 4-year-old mares from 1993 to 2004 were analyzed. Data from a mare were utilized only if she completed all exercises successfully. There were 396 records from 3-year-old and 233 records from 4-year-old mares, respectively, and 69 mares were tested at both ages.

Data were analysed using the SAS (SAS INSTITUT, 1999) software package. Multivariate analysis of variance (MANOVA), which is the generalization of analysis of variance for multivariate cases, was used to compare records from the two ages. Relationships among traits were examined using principal component analysis. This 
method was used to determine the relative contribution of factors to total variation of the traits and which traits belong to the each identified factor. Eigenvalues of factors show the contribution of each component to total variation. During the evaluation, factors were rotated with Varimax rotation of H. F. Kaiser. The aim of the Varimax method is to maximize the sum of variances of $a_{i j}^{2}$ quadratic weights (SVÁB, 1979). The rotation method identifies what is thought to be a valid and biologically reasonable solution, because it accounts for the correlation among background variables. In this case, background variables are not independent from one another. The 'eigenvalue 1.0 criterion', whereby only components with eigenvalues exceeding unity were considered significant and hence retained in the analysis, was used (SZELÉNYI, 1993). Cluster analysis we used for visual representation of the grouping of traits (PODNAI, 1997).

\section{Results \\ Means and standard deviations}

Table 1 shows the mean and standard deviation for each of the recorded traits. In consideration of differences among traits in the range of possible scores, type, head and frame had higher means than other conformation traits in both age-groups. Standard deviations were high in the cases of neck, hindlimbs and impulsion and elasticity of movement. High standard deviations also existed for walk and canter, but the standard deviation for trot was relatively low.

Table 1

Means and standard deviations of traits evaluated in 3-year-old $(n=396)$ and 4-year-old ( $n=233)$ mares (Mittelwerte und Standardabweichung erfasster Merkmale 3- bzw. 4- jähriger Stuten)

\begin{tabular}{lcccc}
\hline \multirow{2}{*}{ Trait } & \multicolumn{2}{c}{ 3-year-old mares } & \multicolumn{2}{c}{ 4-year-old mares } \\
& Mean & St. dev. & Mean & St. dev. \\
\hline Type & 4.73 & 0.879 & 4.71 & 0.931 \\
Head & 6.34 & 0.944 & 6.36 & 0.994 \\
Neck & 8.52 & 1.236 & 8.31 & 1.206 \\
Saddle region & 7.72 & 1.181 & 7.53 & 1.144 \\
Frame & 6.22 & 1.040 & 6.22 & 1.131 \\
Forelimbs & 8.41 & 1.171 & 8.14 & 1.121 \\
Hindlimbs & 8.05 & 1.239 & 7.86 & 1.207 \\
Regularity of movement & 7.56 & 1.064 & 7.54 & 1.083 \\
Impulsion and elasticity of movement & 8.05 & 1.454 & 7.73 & 1.342 \\
Overall impression & 7.17 & 0.963 & 7.00 & 0.883 \\
\hline Jumping style & 7.18 & 0.965 & 7.36 & 1.116 \\
Jumping ability-sense of distance & 7.11 & 1.130 & 7.42 & 1.262 \\
Observation during training & 6.87 & 0.791 & 7.02 & 0.820 \\
\hline Walk & 6.83 & 1.057 & 6.78 & 1.111 \\
Trot & 6.32 & 0.868 & 6.33 & 0.824 \\
Canter & 6.79 & 1.025 & 6.96 & 0.973 \\
Overall impression & 6.90 & 0.881 & 6.95 & 0.924 \\
Test rider's score & 7.16 & 1.146 & 7.34 & 1.154 \\
\hline
\end{tabular}

Multivariate analysis of variance

Effect of age at evaluation on the results of mare tests was estimated with multivariate analysis of variance. Age groups differed $(\mathrm{P}<0.05)$ for the following traits: neck, saddle region, forelimbs, impulsion and elasticity of movement, overall impression, jumping style, jumping ability-sense of distance, observation during training and canter. Scores for conformation traits were lower in the older group. 


\section{Correlation analysis}

Correlations among traits are shown in Table 2 and Table 3 for 3-year and 4-year-old test data, respectively.

From the evaluation of data recorded on 3-year-old mares $(n=396)$, low correlations $(\mathrm{P}<0.01)$ were found among conformation traits and free jumping traits. Correlations among conformation traits and movement analysis traits were in the range of 0.17 to $0.48(\mathrm{P}<0.01)$. Higher correlation coefficients were found between overall impression and conformation scores. Free jumping performance and movement analysis traits were lowly correlated. Correlations between jumping style and walk and trot were not significant. High correlations were found between type and head and between type and frame. There were low to moderate correlations among other conformation traits. Within free jumping performance traits, there was a high correlation $(\mathrm{P}<0.01)$ between jumping style and jumping ability-sense of distance. High correlations $(\mathrm{P}<0.01)$ also were found between canter and overall impression through the evaluation of movement analysis traits. Low and moderate correlations were observed among other movement components.

Significant correlations did not exist among conformation traits and observation during training except overall impression for 4-year-old mares $(n=233)$. Correlations were not significant among walk and free jumping performance traits. Low and moderate, significant $(\mathrm{P}<0.01$, or 0.05$)$ correlations were found among further conformation and movement analysis traits. Correlations among free jumping and movement analysis traits were in the range of 0.15 and $0.39(\mathrm{P}<0.01)$. High correlations were found between type and head and between type and frame. Correlations among further conformation traits were moderate to low $(\mathrm{P}<0.01)$. Evaluation of free jumping performance was highly correlated with jumping style and jumping ability-sense of distance. Correlations among movement analysis traits were in the range of 0.41 to $0.61(\mathrm{P}<0.01)$.

\section{Principal component matrix}

Using principal component analysis, traits were aggregated into groups. In this analysis, only traits with correlation coefficients $\geq 0.5$ were used in the factor description.

After Varimax rotation, 9 factors with the ratio of variance equal to 80.935 were identified based on test results of 3-year-old mares. Factor pattern coefficients of the rotated factors are shown in Table 4, with factors ordered according to the proportion of variation that they account for. These coefficients show the relative contribution of traits to the particular factor. Factor I was determined by some conformation traits (a type, head, saddle region, frame) and by overall impression. Factor II was related to movement analysis traits, determined by trot, canter, overall impression and test rider's score. Factor III was determined by the free jumping performance traits, jumping style and jumping ability-sense of distance. Factor IV was determined primarily by a single trait, impulsion and elasticity of movement. Factor $\mathrm{V}$ was determined primarily by walk, from movement analysis. Factor VI was affected primarily by the forelimbs trait from conformation; whereas Factor VII was dependent upon hindlimbs. Factor VIII was influenced primarily by the neck trait from conformation analysis. Factor IX was dependent upon observation during training from free jumping performance. 
Table 2

Correlations among traits evaluated on 3-year-old mares (Korrelationskoeffizienten zwischen Merkmalen 3-jähriger Stuten)

\begin{tabular}{|c|c|c|c|c|c|c|c|c|c|c|c|c|c|c|c|c|c|c|c|}
\hline Trai & & 1 & 2 & 3 & 4 & 5 & 6 & 7 & 8 & 9 & 10 & 11 & 12 & 13 & 14 & 15 & 16 & 17 & 18 \\
\hline 1 & Type & 1 & & & & & & & & & & & & & & & & & \\
\hline 2 & Head & $0.71^{* *}$ & 1 & & & & & & & & & & & & & & & & \\
\hline 3 & Neck & $0.47^{* *}$ & $0.41^{* *}$ & 1 & & & & & & & & & & & & & & & \\
\hline 4 & Saddle region & $0.61^{* *}$ & $0.49^{* *}$ & $0.47^{* *}$ & 1 & & & & & & & & & & & & & & \\
\hline 5 & Frame & $0.77^{* *}$ & $0.52^{* *}$ & $0.44^{* *}$ & $0.60^{* *}$ & 1 & & & & & & & & & & & & & \\
\hline 6 & Forelimbs & $0.49^{* *}$ & $0.35^{* *}$ & $0.36^{* *}$ & $0.49^{* *}$ & $0.46^{* *}$ & 1 & & & & & & & & & & & & \\
\hline 7 & Hindlimbs & $0.42^{* *}$ & $0.28^{* *}$ & $0.32^{* *}$ & $0.36^{* *}$ & $0.42^{* *}$ & $0.44^{* *}$ & 1 & & & & & & & & & & & \\
\hline 8 & $\begin{array}{l}\text { Regularity of } \\
\text { movement }\end{array}$ & $0.56^{* *}$ & $0.48^{* *}$ & $0.33^{* *}$ & $0.41^{* *}$ & $0.49^{* *}$ & $0.41^{* *}$ & $0.40^{* *}$ & 1 & & & & & & & & & & \\
\hline 9 & $\begin{array}{l}\text { Impulsion and } \\
\text { elasticity of } \\
\text { movement }\end{array}$ & $0.40^{* *}$ & $0.24^{* *}$ & $0.39^{* *}$ & $0.23^{* *}$ & $0.33^{* *}$ & $0.30^{* *}$ & $0.34^{* *}$ & $0.45^{* *}$ & 1 & & & & & & & & & \\
\hline 10 & $\begin{array}{l}\text { Overall } \\
\text { impression }\end{array}$ & $0.69^{* *}$ & $0.51^{* *}$ & $0.56^{* *}$ & $0.56^{* *}$ & $0.64^{* *}$ & $0.53^{* *}$ & $0.47^{* *}$ & $0.48^{* *}$ & $0.65^{* *}$ & 1 & & & & & & & & \\
\hline 11 & Jumping style & $0.16^{* *}$ & $0.14^{* *}$ & $0.23^{* *}$ & $0.20^{* *}$ & $0.24^{* *}$ & $0.15^{* *}$ & $0.22^{* *}$ & $0.18^{* *}$ & $0.22^{* *}$ & $0.29^{* *}$ & 1 & & & & & & & \\
\hline 12 & $\begin{array}{l}\text { Jumping ability- } \\
\text { sense of distance }\end{array}$ & $0.29^{* *}$ & $0.22^{* *}$ & $0.28^{* *}$ & $0.31^{* *}$ & $0.34^{* *}$ & $0.22^{* *}$ & $0.25^{* *}$ & $0.31^{* *}$ & $0.30^{* *}$ & $0.40^{* *}$ & $0.77^{* *}$ & 1 & & & & & & \\
\hline 13 & $\begin{array}{l}\text { Observation } \\
\text { during training }\end{array}$ & $0.23^{* *}$ & $0.17^{* *}$ & $0.18^{* *}$ & $0.17^{* *}$ & $0.25^{* *}$ & $0.12^{*}$ & $0.15^{* *}$ & $0.14^{* *}$ & $0.24^{* *}$ & $0.29^{* *}$ & $0.31^{* *}$ & $0.52^{* *}$ & 1 & & & & & \\
\hline 14 & Walk & $0.37^{* *}$ & $0.38_{* *}^{* *}$ & $0.18^{* * *}$ & $0.31_{* *}^{* *}$ & $0.34^{* * *}$ & $0.24_{* *}^{* *}$ & $0.21_{* * *}^{* *}$ & $0.39^{* *}$ & $0.37^{* *}$ & $0.36_{* *}^{* *}$ & 0.07 & $0.22_{* *}^{* *}$ & $0.18^{* *}$ & 1 & & & & \\
\hline 15 & Trot & $0.29^{* *}$ & $0.19^{* *}$ & $0.23^{* *}$ & $0.20^{* *}$ & $0.27^{* *}$ & $0.18^{* *}$ & $0.16^{* *}$ & $0.22^{* *}$ & $0.47^{* *}$ & $0.38^{* *}$ & 0.08 & $0.21_{* *}^{* *}$ & $0.22^{* *}$ & $0.36^{* *}$ & & & & \\
\hline 16 & Canter & $0.36^{* *}$ & $0.29^{* *}$ & $0.30^{* *}$ & $0.28^{* *}$ & $0.36^{* *}$ & $0.21^{* *}$ & $0.17^{* *}$ & $0.29^{* *}$ & $0.39^{* *}$ & $0.38^{* *}$ & $0.21^{* *}$ & $0.31^{* *}$ & $0.30^{* *}$ & $0.39^{* * *}$ & $0.58^{* *}$ & 1 & & \\
\hline 17 & $\begin{array}{l}\text { Overall } \\
\text { impression }\end{array}$ & $0.48^{* *}$ & $0.42^{* *}$ & $0.34^{* *}$ & $0.42^{* *}$ & $0.45^{* *}$ & $0.32^{* *}$ & $0.26^{* *}$ & $0.43^{* *}$ & $0.41^{* *}$ & $0.47^{* *}$ & $0.19^{* *}$ & $0.36^{* *}$ & $0.26^{* *}$ & $0.55^{* *}$ & $0.56^{* *}$ & $0.70^{* *}$ & 1 & \\
\hline 18 & Test rider's score & $0.35^{* *}$ & $0.28^{* *}$ & $0.28^{* *}$ & $0.30^{* *}$ & $0.37^{* *}$ & $0.19^{* *}$ & $0.20^{* *}$ & $0.29^{* *}$ & $0.29^{* *}$ & $0.36^{* *}$ & $0.26^{* *}$ & $0.34^{* *}$ & $0.28^{* *}$ & $0.40^{* * *}$ & $0.38^{* *}$ & $0.49^{* *}$ & $0.62^{* *}$ & 1 \\
\hline
\end{tabular}


Table 3

Correlations among traits evaluated on 4-year-old mares (Korrelationskoeffizienten zwischen Merkmalen 4- jähriger Stuten)

\begin{tabular}{|c|c|c|c|c|c|c|c|c|c|c|c|c|c|c|c|c|c|c|c|}
\hline Trait & & 1 & 2 & 3 & 4 & 5 & 6 & 7 & 8 & 9 & 10 & 11 & 12 & 13 & 14 & 15 & 16 & 17 & 18 \\
\hline 1 & Type & 1 & & & & & & & & & & & & & & & & & \\
\hline 2 & Head & $0.76^{* *}$ & 1 & & & & & & & & & & & & & & & & \\
\hline 3 & Neck & $0.36^{* *}$ & $0.25^{* *}$ & 1 & & & & & & & & & & & & & & & \\
\hline 4 & Saddle region & $0.66^{* *}$ & $0.49^{* *}$ & $0.48^{* *}$ & 1 & & & & & & & & & & & & & & \\
\hline 5 & Frame & $0.80^{* *}$ & $0.57^{* *}$ & $0.41^{* *}$ & $0.61^{* *}$ & 1 & & & & & & & & & & & & & \\
\hline 6 & Forelimbs & $0.44^{* *}$ & $0.34^{* *}$ & $0.49^{* *}$ & $0.49^{* *}$ & $0.46^{* *}$ & 1 & & & & & & & & & & & & \\
\hline 7 & Hindlimbs & $0.38^{* *}$ & $0.28^{* *}$ & $0.44^{* *}$ & $0.39^{* *}$ & $0.42^{* *}$ & $0.48^{* *}$ & 1 & & & & & & & & & & & \\
\hline 8 & $\begin{array}{l}\text { Regularity of } \\
\text { movement }\end{array}$ & $0.64^{* *}$ & $0.50^{* *}$ & $0.32^{* *}$ & $0.56^{* *}$ & $0.64^{* *}$ & $0.52^{* *}$ & $0.42^{* *}$ & 1 & & & & & & & & & & \\
\hline 9 & $\begin{array}{l}\text { Impulsion and } \\
\text { elasticity of } \\
\text { movement }\end{array}$ & $0.31^{* *}$ & $0.23^{* *}$ & $0.45^{* *}$ & $0.28^{* *}$ & $0.33^{* *}$ & $0.46^{* *}$ & $0.39^{* *}$ & $0.46^{* *}$ & 1 & & & & & & & & & \\
\hline 10 & $\begin{array}{l}\text { Overall } \\
\text { impression }\end{array}$ & $0.69^{* *}$ & $0.55^{* *}$ & $0.58^{* *}$ & $0.56^{* *}$ & $0.64^{* *}$ & $0.56^{* *}$ & $0.54^{* *}$ & $0.48^{* *}$ & $0.61^{* *}$ & 1 & & & & & & & & \\
\hline 11 & Jumping style & $0.15^{*}$ & 0.03 & $0.17^{* *}$ & $0.21^{* *}$ & 0.09 & $0.27^{* *}$ & $0.21^{* *}$ & $0.21^{* *}$ & $0.17^{* *}$ & $0.13^{*}$ & 1 & & & & & & & \\
\hline 12 & $\begin{array}{l}\text { Jumping ability- } \\
\text { sense of distance }\end{array}$ & $0.23^{* *}$ & 0.09 & 0.08 & $0.22^{* *}$ & $0.15^{*}$ & $0.26^{* *}$ & $0.14^{*}$ & $0.28^{* *}$ & $0.18^{* *}$ & $0.15^{*}$ & $0.84^{* *}$ & 1 & & & & & & \\
\hline 13 & $\begin{array}{l}\text { Observation } \\
\text { during training }\end{array}$ & 0.08 & 0.06 & 0.06 & 0.08 & 0.07 & 0.09 & 0.02 & 0.09 & 0.09 & $0.11^{*}$ & $0.47^{* *}$ & $0.55^{* *}$ & 1 & & & & & \\
\hline 14 & Walk & $0.31^{* *}$ & $0.31^{* *}$ & $0.11^{*}$ & $0.25^{* *}$ & $0.27^{* *}$ & $0.22^{* *}$ & $0.12^{*}$ & $0.41^{* *}$ & $0.27^{* *}$ & $0.24^{* *}$ & 0.05 & 0.11 & 0.10 & 1 & & & & \\
\hline 15 & Trot & $0.24^{* *}$ & $0.21_{* * *}^{* *}$ & $0.19^{* *}$ & $0.17^{* *}$ & $0.27^{* *}$ & $0.23^{* *}$ & $0.12^{*}$ & $0.21^{* *}$ & $0.46^{* *}$ & $0.35^{* *}$ & $0.22^{* *}$ & $0.19^{* *}$ & $0.18^{* *}$ & $0.48^{* *}$ & 1 & & & \\
\hline 16 & Canter & $0.36^{* *}$ & $0.28^{* *}$ & $0.12^{*}$ & $0.35^{* *}$ & $0.31^{* *}$ & $0.29^{* *}$ & $0.14^{*}$ & $0.40^{* *}$ & $0.32^{* *}$ & $0.30^{* *}$ & $0.29^{* *}$ & $0.32^{* *}$ & $0.32^{* *}$ & $0.46^{* *}$ & $0.52^{* *}$ & 1 & & \\
\hline 17 & $\begin{array}{l}\text { Overall } \\
\text { impression }\end{array}$ & $0.48^{* *}$ & $0.36^{* *}$ & $0.25^{* *}$ & $0.39^{* *}$ & $0.48^{* *}$ & $0.36^{* *}$ & $0.22^{* *}$ & $0.41^{* *}$ & $0.29^{* *}$ & $0.41^{* *}$ & $0.24^{* *}$ & $0.25^{* *}$ & $0.15^{* *}$ & $0.57^{* *}$ & $0.58^{* *}$ & $0.57^{* *}$ & 1 & \\
\hline 18 & Test rider's score & $0.36^{* *}$ & $0.25^{* *}$ & $0.14^{*}$ & $0.35^{* *}$ & $0.34^{* *}$ & $0.22^{* *}$ & $0.18^{* *}$ & $0.37^{* *}$ & $0.23^{* *}$ & $0.35^{* *}$ & $0.34^{* *}$ & $0.39^{* *}$ & $0.30^{* *}$ & $0.41^{* *}$ & $0.44^{* *}$ & $0.53^{* *}$ & $0.61^{* *}$ & 1 \\
\hline
\end{tabular}

${ }^{*}=\mathrm{P}<0.05,{ }^{* *}=\mathrm{P}<0.01$ 
Table 4

Eigenvalues and share of total variance, factors and factor loadings after rotation based on analysis of data from 3-year-old mares (Eigenwerte und deren Varianz der Faktoren und Faktorzuordnung nach der Rotation auf der Grundlage von Daten 3-jähriger Stuten)

\begin{tabular}{|c|c|c|c|c|c|c|c|c|c|}
\hline Trait & $\begin{array}{l}\text { Factor } \\
\text { I. }\end{array}$ & $\begin{array}{l}\text { Factor } \\
\text { II. }\end{array}$ & $\begin{array}{l}\text { Factor } \\
\text { III. }\end{array}$ & $\begin{array}{l}\text { Factor } \\
\text { IV. }\end{array}$ & $\begin{array}{c}\text { Factor } \\
\text { V. }\end{array}$ & $\begin{array}{l}\text { Factor } \\
\text { VI. }\end{array}$ & $\begin{array}{l}\text { Factor } \\
\text { VII. }\end{array}$ & $\begin{array}{l}\text { Factor } \\
\text { VIII. }\end{array}$ & $\begin{array}{l}\text { Factor } \\
\text { IX. }\end{array}$ \\
\hline Eigenvalues & 3.269 & 2.527 & 1.897 & 1.429 & 1.199 & 1.096 & 1.080 & 1.048 & 1.022 \\
\hline $\begin{array}{l}\text { Variance of } \\
\text { eigenvalues \% }\end{array}$ & 18.164 & 14.040 & 10.541 & 7.940 & 6.659 & 6.090 & 5.999 & 5.822 & 5.680 \\
\hline Type & 0.846 & 0.188 & 0.042 & 0.170 & 0.069 & 0.114 & 0.138 & 0.092 & 0.082 \\
\hline Head & 0.722 & 0.125 & 0.016 & -0.001 & 0.143 & 0.010 & -0.086 & 0.174 & 0.071 \\
\hline Neck & 0.321 & 0.135 & 0.114 & 0.220 & -0.025 & 0.148 & 0.061 & 0.842 & 0.047 \\
\hline Saddle region & 0.669 & 0.117 & 0.153 & -0.026 & 0.202 & 0.394 & 0.075 & 0.248 & -0.012 \\
\hline Frame & 0.807 & 0.200 & 0.152 & 0.113 & 0.062 & 0.105 & 0.250 & 0.037 & 0.062 \\
\hline Forelimbs & 0.299 & 0.109 & 0.067 & 0.093 & 0.065 & 0.837 & 0.217 & 0.117 & 0.020 \\
\hline Hindlimbs & 0.248 & 0.035 & 0.096 & 0.183 & 0.009 & 0.228 & 0.836 & 0.054 & 0.051 \\
\hline $\begin{array}{l}\text { Regularity of } \\
\text { movement }\end{array}$ & 0.335 & 0.144 & 0.136 & 0.197 & 0.169 & 0.149 & 0.223 & 0.061 & -0.015 \\
\hline $\begin{array}{l}\text { Impulsion and } \\
\text { elasticity of } \\
\text { movement }\end{array}$ & 0.114 & 0.243 & 0.128 & 0.825 & 0.164 & 0.038 & 0.155 & 0.180 & 0.069 \\
\hline Overall impression & 0.573 & 0.171 & 0.183 & 0.535 & 0.116 & 0.208 & 0.198 & 0.252 & 0.096 \\
\hline Jumping style & 0.076 & 0.062 & 0.946 & 0.062 & -0.016 & 0.019 & 0.072 & 0.074 & 0.034 \\
\hline $\begin{array}{l}\text { Jumping ability- } \\
\text { sense of distance }\end{array}$ & 0.158 & 0.154 & 0.852 & 0.100 & 0.090 & 0.082 & 0.043 & 0.048 & 0.288 \\
\hline $\begin{array}{l}\text { Observation } \\
\text { during training }\end{array}$ & 0.109 & 0.159 & 0.251 & 0.077 & 0.062 & 0.013 & 0.048 & 0.041 & 0.935 \\
\hline Walk & 0.207 & 0.247 & 0.010 & 0.214 & 0.845 & 0.113 & -0.018 & -0.054 & 0.064 \\
\hline Trot & 0.128 & 0.760 & -0.004 & 0.446 & 0.025 & 0.065 & -0.019 & -0.088 & 0.055 \\
\hline Canter & 0.146 & 0.850 & 0.118 & 0.088 & 0.055 & 0.077 & -0.010 & 0.107 & 0.118 \\
\hline Overall impression & 0.267 & 0.744 & 0.118 & 0.043 & 0.346 & 0.101 & 0.089 & 0.124 & 0.054 \\
\hline Test rider's score & 0.183 & 0.560 & 0.208 & -0.123 & 0.453 & -0.183 & 0.325 & 0.271 & 0.069 \\
\hline
\end{tabular}

After Varimax rotation, 7 factors with ratio of variance of 74.115 were identified from analysis of data from 4-year-old mares. Factor pattern coefficients of the rotated factors are shown in Table 5. Factor I was determined by some conformation traits (type, head, saddle region, frame) and by regularity of movement and overall impression. Factor II was related to movement analysis traits (trot, canter) and by overall impression and test rider's score. Factor III was determined by the free jumping performance traits, jumping style and jumping ability-sense of distance. Factor IV was affected primarily by a single trait, impulsion and elasticity of movement. Factor $\mathrm{V}$ was primarily determined by the neck trait from conformation analysis, Factor VI was determined primarily by hindlimbs and Factor VII was dependent upon walk from movement analysis.

Figure 1 and 2 illustrate the separation of traits among trait groups by cluster analysis of test results of 3-year-old and 4-year-old mares, respectively. 
Table 5

Eigenvalues and share of total variance, factors and factor loadings after rotation based on analysis of data from 4-year-old mares (Eigenwerte und deren Varianz der Faktoren und Faktorenzuordnung nach der Rotation auf der Grundlage von Daten 4-jähriger Stuten)

\begin{tabular}{|c|c|c|c|c|c|c|c|}
\hline Trait & Factor I. & Factor II. & $\begin{array}{l}\text { Factor } \\
\text { III. }\end{array}$ & $\begin{array}{l}\text { Factor } \\
\text { IV. }\end{array}$ & Factor V. & $\begin{array}{l}\text { Factor } \\
\text { VI. }\end{array}$ & $\begin{array}{l}\text { Factor } \\
\text { VII. }\end{array}$ \\
\hline Eigenvalues & 3.631 & 2.426 & 2.063 & 1.474 & 1.451 & 1.168 & 1.128 \\
\hline $\begin{array}{l}\text { Variance of eigenvalues } \\
\%\end{array}$ & 20.172 & 13.479 & 11.463 & 8.190 & 8.059 & 6.487 & 6.265 \\
\hline Type & 0.904 & 0.191 & 0.099 & 0.083 & 0.125 & 0.091 & 0.062 \\
\hline Head & 0.885 & 0.028 & -0.013 & 0.048 & -0.026 & 0.059 & 0.190 \\
\hline Neck & 0.211 & 0.014 & 0.044 & 0.233 & 0.873 & 0.180 & 0.043 \\
\hline Saddle region & 0.573 & 0.262 & 0.116 & -0.048 & 0.480 & 0.052 & -0.015 \\
\hline Frame & 0.772 & 0.240 & 0.026 & 0.116 & 0.221 & 0.156 & 0.016 \\
\hline Forelimbs & 0.302 & 0.054 & 0.204 & 0.201 & 0.343 & 0.323 & 0.109 \\
\hline Hindlimbs & 0.249 & 0.052 & 0.086 & 0.151 & 0.188 & 0.896 & 0.014 \\
\hline Regularity of movement & 0.542 & 0.135 & 0.172 & 0.296 & 0.047 & 0.162 & 0.289 \\
\hline $\begin{array}{l}\text { Impulsion and elasticity } \\
\text { of movement }\end{array}$ & 0.160 & 0.123 & 0.079 & 0.892 & 0.210 & 0.159 & 0.091 \\
\hline Overall impression & 0.609 & 0.197 & 0.003 & 0.425 & 0.381 & 0.313 & -0.041 \\
\hline Jumping style & 0.004 & 0.153 & 0.931 & 0.042 & 0.095 & 0.086 & -0.020 \\
\hline $\begin{array}{l}\text { Jumping ability-sense } \\
\text { of distance }\end{array}$ & 0.101 & 0.138 & 0.913 & 0.064 & -0.017 & 0.015 & 0.025 \\
\hline $\begin{array}{l}\text { Observation during } \\
\text { training }\end{array}$ & 0.023 & 0.131 & 0.384 & 0.018 & 0.032 & -0.011 & 0.038 \\
\hline Walk & 0.168 & 0.366 & -0.025 & 0.117 & 0.027 & 0.009 & 0.873 \\
\hline Trot & 0.113 & 0.603 & 0.113 & 0.464 & 0.048 & -0.059 & 0.259 \\
\hline Canter & 0.178 & 0.703 & 0.095 & 0.177 & -0.092 & -0.069 & 0.099 \\
\hline Overall impression & 0.317 & 0.717 & 0.145 & 0.021 & 0.150 & 0.072 & 0.355 \\
\hline Test rider's score & 0.185 & 0.787 & 0.248 & 0.044 & 0.066 & 0.137 & 0.111 \\
\hline
\end{tabular}

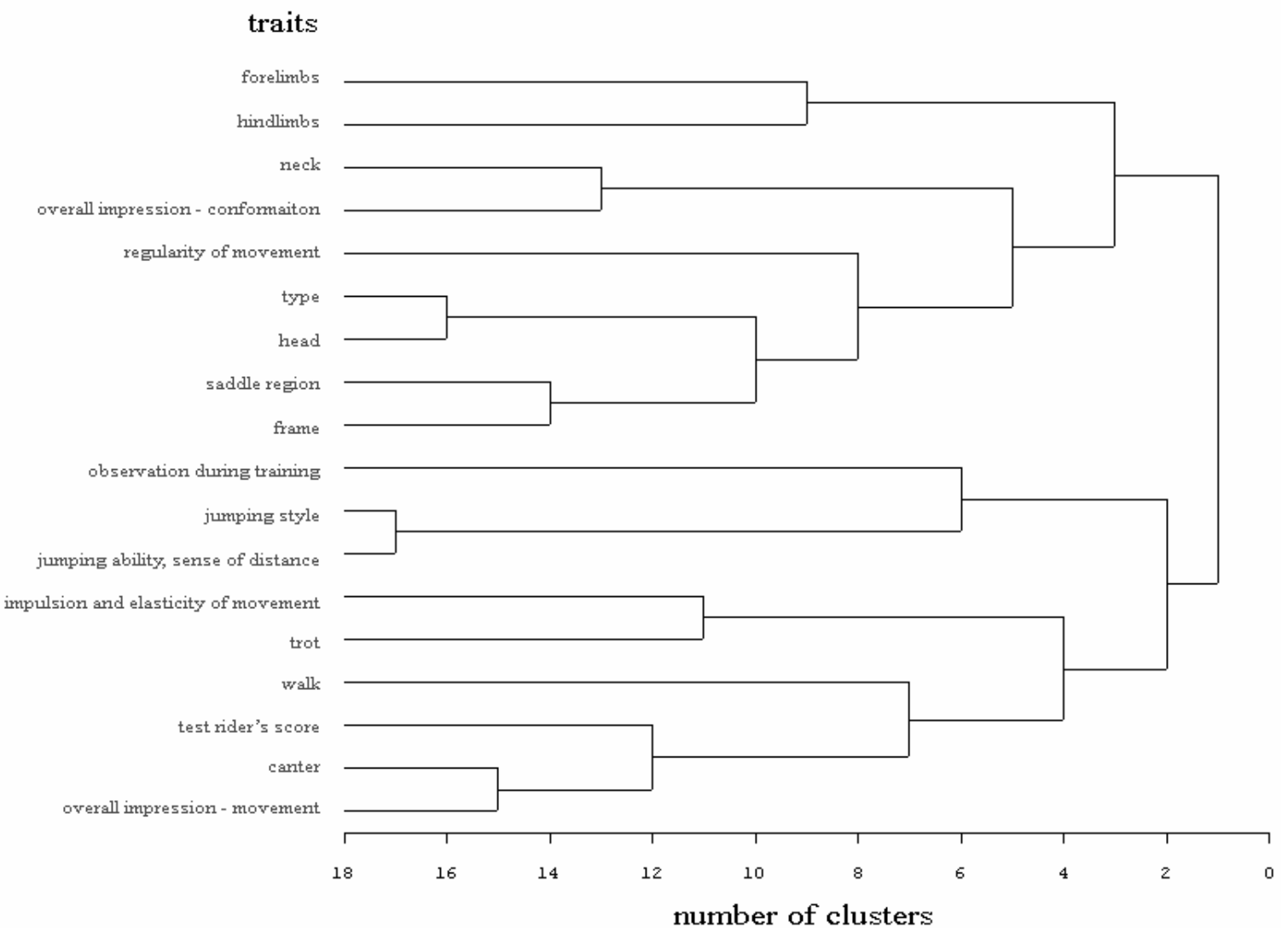

Fig. 1: Dendogram of traits based on SPT data on mares measured at 3 years of age (Dendogramm von Merkmalen auf der Grundlage von SPT Daten von 3-jährigen Stuten) 


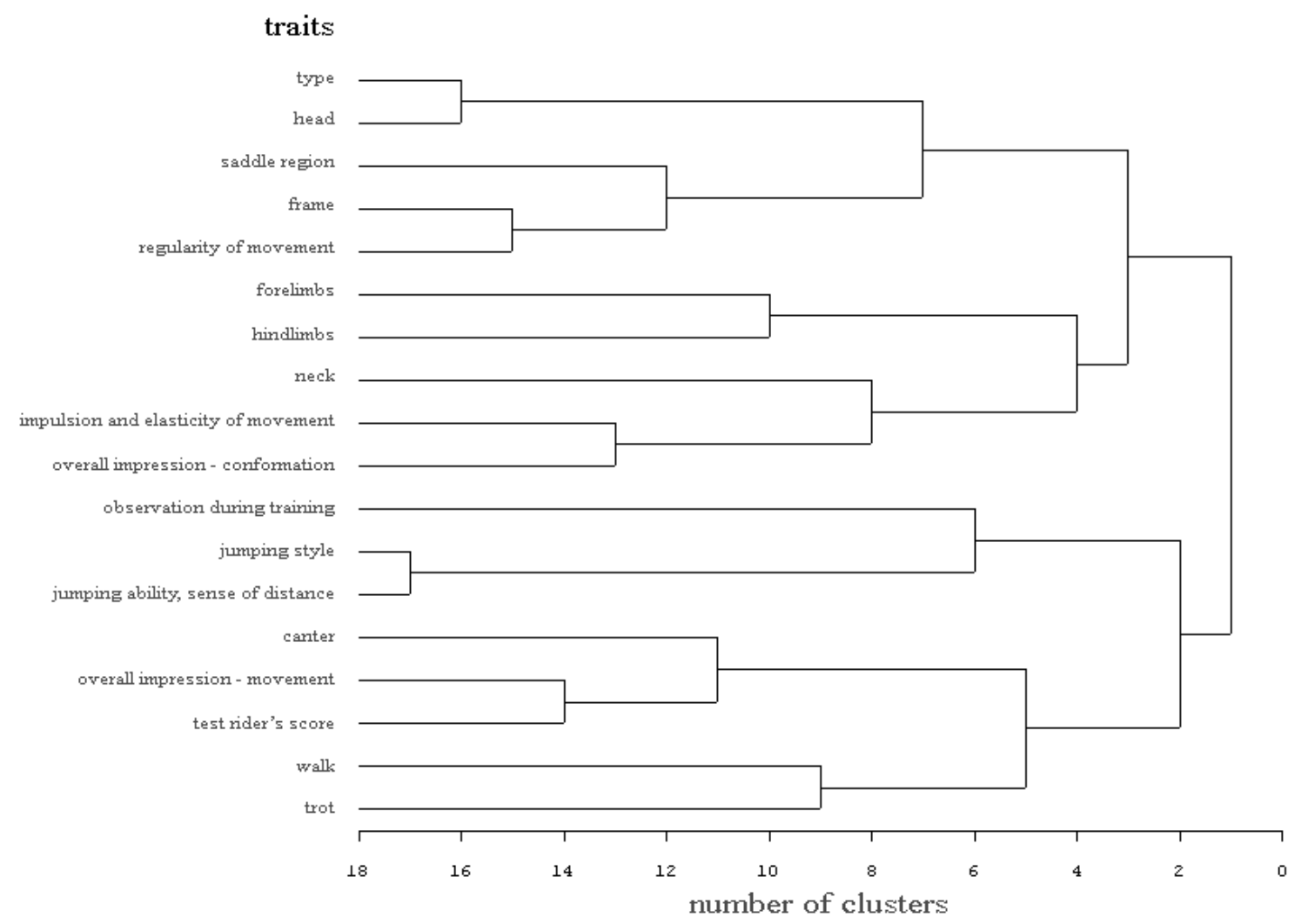

Fig. 2: Dendogram of traits based on SPT data on mares measured at 4 years of age (Dendogramm von Merkmalen auf der Grundlage von SPT Daten 4-jähriger Stuten)

\section{Discussion}

\section{Means and standard deviations}

Means of scores for gait traits (walk, trot and canter) are lower than those reported by HUIZINGA et al. (1990) in a Dutch Warmblood horse population. Such differences could result from differences in skills of the animals or their training. Standard deviations were larger for walk and canter scores but smaller for trot score in the Dutch than in the Hungarian population. This could reflect either differences between population in actual heterogeneity, differential utilization by scorers of the point scale for the traits or both effects.

\section{Multivariate analysis of variance}

Scores for traits of free jumping performance and movement analysis were higher in 3than in 4-year-old mares, which could be attributed to training and experience of the horses. Age, therefore, should be taken into account during evaluation of test results. Ignoring age could provide an advantage in the case of conformation traits but a disadvantage in the case of free jumping performance and movement analysis traits for 3-year-old mares.

\section{Correlation analysis}

The low and in some cases non-significant correlations between conformation components and performance traits suggest that recorded traits have been appropriately assigned to these groups. Low correlations among the traits of free 
jumping performance and movement analysis show the differentiation of these performance traits clearly in both age groups also.

\section{Principal component matrix}

Principal component analysis has been used in Hungary for other species as well. TÖZSÉR et al. (2000) used this method for investigation of conformation traits of weaned Charolais calves. VISSER et al. used principal component analysis to quantify associations between behavioural variables and temperament in young horses (2001) and the association between show-jumping performance and personality traits (2003). SEAMAN et al. (2002) analyzed horse temperament by principal component analysis. As shown in Tables 4 and 5, age groups differ in the manner in which traits are assigned to different trait groups. Similar conclusions were drawn from productmoment correlation analysis. In particular, conformation traits can be aggregated into different sub-groups depending on age at which the mares were evaluated.

Using cluster analysis to classify traits to trait groups separately for each age group helped to clarify the separation of traits. The trait, "impulsion and elasticity of movement" from conformation judgement, can be classified as a movement judgement trait based on test results from 3-year-old mares but can be classified to conformation traits, especially to the trait, overall impression, based on records of 4-year-old mares. This difference could be attributed to the training of the horse, because impulsion and elasticity of movement is similar to movement analysis traits among 3-year-old mares but is more similar to free jumping performance traits for 4-year-old mares.

\section{Acknowledgements}

This work was made possible by the financial support of the "4/057/2004 NKFP" research project in a close cooperation with The Association of Hungarian Horse Breeders and Horse Organization and The Association of Hungarian Sporthorse Breeders (MSLT). The Association of Hungarian Sporthorse Breeders, and especially Zoltán Prutkay, are gratefully acknowledged for providing the data set for the study. The authors express great appreciation to Dr. William D. Hohenboken for his helpful comments on the manuscript.

\section{References}

BUGISLAUS, A.-E.; ROEHE, R.; UPHAUS, H.; KALM, E.:

Development of genetic models for estimation of racing performances in German thoroughbreds. Arch. Tierz., Dummerstorf 47 (2004), 505-516

DIETL, G.; HOFFMANN, S.; ALBRECHT, S.:

Parameter und Trends der Stutbuchaufnahme des Mecklenburger Warmblut Pferdes. Arch. Tierz., Dummerstorf 47 (2004), 107-117

DIETL, G.; HOFFMANN, S.; REINSCH, N.:

Impact of trainer and judges in the mare performance test of Warmblood horses. Arch. Tierz., Dummerstorf 48 (2005), 113-120

HUIZINGA, H.A.; BOUCAMP, M.; SMOLDERS, G.:

Estimated parameters of field performance testing of mares from the Dutch Warmblood riding horse population. Livestock Production Science 26 (1990), 291-299

KOENEN, E.C.P.:

Prüfungsverfahren und Zuchtwertschätzung für Sportpferde im internationalen Vergleich. Arch. Tierz., Dummerstorf 45 (2002) Sonderheft, 38-44

LENGERKEN, G. v.; SCHWARK, H.-J.:

Exterieur und Leistungen in der Pferdezucht - Alleskönner oder Spezialisten. Arch. Tierz., 45 (2002)

Sonderheft, 68-79 


\section{MIHÓK, S.:}

Selection of sport and racehorses (A sport- és a versenyló szelekciója); Acta Agraria Debreceniensis 18 (2005), 3-10

MSLT

Breeding regulation of Hungarian Sport Horse Breeders (A Magyar Sportlótenyésztők Országos Egyesületének Tenyésztési Szabályzata) (2000)

PETZOLD, P.

Die Anwendung des BLUP-Verfahrens zur Zuchtwertschätzung in der Pferdezucht Europas. Arch.

PODNAI, J.:

Tierz., Dummerstorf 34 (1991), 293-297

Introduction to the secrets of multivariate biological data analysis (Bevezetés a többváltozós biológiai

SAS Institut Inc. adatelemzés rejtelmeibe), Scientia Publishing, Budapest (1997) 412

SAS /STAT Software Release 8.2., Cary, NC, USA (1999)

SCHWARK, H.-J.; PETZOLD, P.; KARWATH, M.:

Untersuchungen zur Objektivität und zur züchterischen Effektivität der stationären Eigenleistungsprüfungen von Hengsten der Reitpferderassen. Arch. Tierz., Dummerstorf 35 (1992), 97-106

SEAMAN, S.C.; DAVIDSON, H.P.B.; WARAN, N.K.:

How reliable is temperament assessment in the domestic horse (Equus caballus)? Applied Animal SVÁB, J.: Behaviour Science 78 (2002), 175-191

Multivariate methods in biometry (Többváltozós módszerek a biometriában), Agricultural Publishing, Budapest, (1979), 45-78

SZELÉNYI, L.:

Multivariate methods. In: Harnos Zs, Biometrical methods and its application with MINITAB software package. (Többváltozós módszerek. In: Harnos Zs, Biometriai módszerek és alkalmazásaik MINITAB programcsomaggal.) Gödöllő, (1993), 163-184

TÖZSÉR, J.; DOMOKOS, Z.; ALFÖLDI, L.; SVÁB, L.; MILICZKI, L.:

The relationship of body measurements and conformation traits in Charolais weaned bull calves (Charolais fajtájú választott bikaborjak testméretének és küllemi tulajdonságainak összefüggése), Hungarian Journal of Animal Production 49 (2000) 4, 301-312

VISSER, E.K.; van REENEN, C.G.; HOPSTER, H.; SCHILDER, M.B.H.; KNAAP, J.H.; BARNEVELD, A.;

BLOKHUIS, H.J.:

Quantifying aspects of young horses' temperament: consistency of behavioural variables, Applied Animal Behaviour Science 74 (2001), 241-258

VISSER, E.K.; van REENEN, C.G.; ENGEL, B.; SCHILDER, M.B.H.; BARNEVELD, A.; BLOKHUIS, H.J.: The association between performance in show-jumping and personality traits earlier in life. Applied Animal Behaviour Science 82 (2003), 279-295

Received: 2006-06-07

Accepted: 2007-01-08

Authors addresses

JÁNOS POSTA*, PhD student, ISTVÁN KOMLÓSI, associate professor,

SÁNDOR MIHÓK, professor

University of Debrecen, Centre of Agricultural Sciences, Department of Animal Science

Böszörményi út 138.

4032 DEBRECEN, HUNGARY

*Corresponding author, Hungary e-mail: postaj@agr.unideb.hu

Hungarye-mail: komlosi@agr.unideb.hu

Hungarye-mail: mihok@agr.unideb.hu 